\title{
Radioiodine treatment after surgery for differentiated thyroid cancer: a reasonable option
}

\author{
Jérôme Clerc ${ }^{1}$ - Frederik A. Verburg ${ }^{2}$ - Anca M. Avram ${ }^{3}$ - Luca Giovanella ${ }^{4}$. \\ Elif Hindié $^{5}$ - David Taïeb ${ }^{6}$
}

Published online: 17 February 2017

(C) Springer-Verlag Berlin Heidelberg 2017

\section{Introduction}

Until recently, the usual treatment for differentiated thyroid cancers (DTC) with a size $>1.0 \mathrm{~cm}$ undisputedly consisted of total or near-total thyroidectomy followed by radioiodine administration. Observational data support lower recurrence rates after radioiodine treatment (RAI) following surgery [1-3]. In recent years, and especially so since the publication of the 2015 American Thyroid Association (ATA) differentiated thyroid cancer guidelines (DTC) [4], the systematic use of RAI has declined, or at least has been called into question. The decision on whether to give RAI, and if so what ${ }^{131} \mathrm{I}$ activity to administer, increasingly relies upon risk stratification systems (RSS) such as the ATA risk of recurrence system or the DTC-specific survival oriented UICC/AJCC TNM staging system. Each system takes into account the extent of disease at surgery and additional characteristics such as age, histology, and possibly other patient- or

David Taïeb

david.taieb@ap-hm.fr

1 Department of Nuclear Medicine, Cochin University Hospital, Paris 5 René Descartes University, Paris, France

2 Department of Nuclear Medicine, University Hospital Marburg, Marburg, Germany

3 Nuclear Medicine/Radiology, University of Michigan, Ann Arbor, USA

4 Department of Nuclear Medicine and Thyroid Centre, Oncology Institute of Southern Switzerland, Bellinzona, Switzerland

5 Nuclear Medicine, Haut-Lévêque University Hospital, Bordeaux, France

6 Department of Nuclear Medicine, European Center for Research in Medical Imaging, La Timone University Hospital, Aix-Marseille University, 264 rue Saint-Pierre, 13385 Marseille, France treatment-related factors. The ultimate goal of such systems is to predict risk of recurrence (ATA) or of death from DTC (UICC/AJCC) in order to modulate the intensity of primary treatment and subsequent long-term follow-up strategy. Their use theoretically provides a scientific rationale to prescribe or abstain from RAI following surgery, with the aim of avoiding "overtreatment". In the recent DTC guideline update from ATA [4], less than total thyroidectomy is recommended for intrathyroidal tumors $<4.0 \mathrm{~cm}$, which de facto cancels the possibility of using post-operative therapeutic radio-iodine administration. Even in some more advanced situations, avoidance of postoperative therapeutic radioiodine administration is advised for patients considered at low-to-intermediate risk of recurrence according to the current risk stratification model. However, a safe application of this "de-escalation" approach is still hampered by several significant simplifications and approximations, contradictions and most importantly the lack of solid evidence to support the choices made based on stratification systems predicated on limited surgical pathology information. Additionally, the objectives for RAI administration (e.g. remnant ablation, adjuvant 131-I therapy, or 131-I therapy for metastatic disease) are often merged in the recommendations, with implications for the 131-I activity selected and the intended outcome of RAI treatment. Finally, some recommendations are based on studies with too short follow-up periods ( $<10-15$ years) to assess reliably diseasefree survival estimates for most patients [5].

\section{Are there limitations in the current stratification systems used for guiding RAI therapeutic decision-making for DTC patients?}

Several limitations in the current stratification systems impede the effective use of RAI in the patients that may benefit from this treatment. Thyroid cancer is usually a well-differentiated, 
slowly growing disease with a very low disease-specific mortality rate for local-regional disease after complete initial therapy (5-year survival $99.9 \%$ for localized disease, and $97.8 \%$ for regional metastatic disease); however, distant metastatic disease is associated with significantly worse prognosis (5-year survival 55.3\%) (http://seer.cancer.gov/statfacts/html/thyro. $\mathrm{html}$ ). Therefore, staging and stratification systems predicated on "hard oncological criteria" (e.g. overall survival) are not particularly applicable to the majority of DTC patients and are little informative to guide decisions about RAI. Therefore, secondary outcome variables such as rates of persistent disease, rates of recurrent disease, medicoeconomic issues, and quality of life outcomes, should be taken into consideration as well.

One of the most concerning issues in the 2015 ATA Guidelines [4] is the modification of the 2009 ATA risk stratification [6] to include tumors with extra-thyroidal extension (T3) or associated with lymph nodal (LN) metastases (pN1with $\leq 5 \mathrm{LN}$ involved; all $<0.2 \mathrm{~cm}$ ) in the low-risk category for which RAI ablation is not recommended, based on low recurrence rates reported in published studies that included RAI ablation after total thyroidectomy. The study cited in support of the contention that similar low recurrence rates occur in patients treated with lobectomy alone, and without post-operative RAI, had sub-optimal surveillance (no assessment for distant metastatic disease, nonstimulated $\mathrm{Tg}$ ), and a short follow-up (median 5.1 years) [7]. In addition, a risk stratification predicated solely on surgical pathology information is inherently limited not only by the extent of surgical dissection and the accuracy of histopathology examination, but also by the complete absence of any information regarding the presence of distant metastases. Without a post-therapy ${ }^{131} \mathrm{I}$ scan, or at least a high-quality diagnostic radio-iodine scan to assess regional and distant metastatic disease in the neck compartments unexplored at surgery and in the remainder of the body, staging, and risk stratification systems are abridged of essential information. The principle of precise initial staging is often disregarded in current guidelines, despite the propensity of DTC for local invasion and metastatic spread. Because of its indolent nature and excellent overall survival in localregional disease, DTC appears to be managed rather as a chronic condition with serial follow-up than with curative intent. Decisions to omit ${ }^{131}$ I therapy are made on incomplete staging and risk stratification, despite well-established evidence for improved recurrence-free survival, disease-specific survival, and overall survival for patients who received ${ }^{131} \mathrm{I}$ therapy postoperatively $[2,8,9]$. The strategy of surveillance without ablation based on the assumption of an indolent disease course cannot be accepted in the context of current evidence demonstrating adverse clinical outcomes when ${ }^{131} \mathrm{I}$ therapy is delayed, both for regional [2] and for distant metastatic disease [10]. Until more prolonged follow-up becomes available to compare the benefits from various strategies, RAI administration remains a reasonable option, especially in the numerous situations where the literature is controversial [2, $11]$.

\section{Is the current risk stratification for disease recurrence internally concordant for decision-making strategies?}

The current risk stratification has several internal incoherencies, which limit its applicability for post-operative RAI decision making. The ATA risk stratification is used to inform on the indication for RAI after thyroidectomy in primary management of DTC as follows: RAI is not recommended in low risk patients, while it should be considered in ATA intermediate risk ones, and is routinely recommended in high risk DTC patients [4]. However, the risk classification, which is used for making the decision to perform or withhold RAI partially depends on the ${ }^{131}$ I image pattern itself, since "no RAI positive metastatic foci outside the thyroid bed" is a selected criterion for the low risk category in this system. In contrast, abnormal RAI uptake in the neck corresponds to intermediate risk. Distant metastatic foci seen using either ${ }^{131} \mathrm{I}$ or ${ }^{18} \mathrm{FDG}$ will correspond to high risk disease. It seems somewhat contradictory to determine the indication for RAI ablation depending on the results of the posttherapy ${ }^{131} \mathrm{I}$ image. To solve this conundrum it would be possible to perform diagnostic pre-ablation ${ }^{123} \mathrm{I}$ or ${ }^{131} \mathrm{I}$ imaging (henceforth abbreviated as Dx WBS), as done by some teams. $[3,12-15]$ The main purpose of pre-ablation imaging is to identify regional and distant metastatic disease which would require higher administered 131-I activities as opposed to low-dose remnant ablation strategy, or identification of bulky regional metastatic disease in unexplored neck compartments, which will direct toward reoperation before ${ }^{131}$ I therapy [12]. In addition to surgical pathology and stimulated thyroglobulin (Tg) levels, the information obtained from DxWBS regarding the presence of iodine-avid regional and/or distant metastases can be used for ${ }^{131}$ I treatment planning $[12,13,16]$. In a study of the University of Michigan Medical Centre, Dx scans identified regional metastases in $35 \%$ of patients, and distant metastases in $8 \%$ of patients referred for post-operative ${ }^{131} \mathrm{I}$ therapy [13] and this information in conjunction with the results of stimulated $\mathrm{Tg}$ levels led to a change in management for $31 \%$ of patients, as compared to proposed management predicated on histopathology findings alone [12]. Visualization of metastatic deposits on DxWBS confirms their capacity to concentrate ${ }^{131} \mathrm{I}$ (iodine-avid disease) and, therefore, their potential to respond to therapeutic ${ }^{131}$ I activity. Location and size of metastatic lesions can be determined on SPECT/CT imaging, thus guiding management: ${ }^{131} \mathrm{I}$ therapy is most effective for smaller metastatic deposits $[17,18]$, whereas for large metastases, surgical debulking before ${ }^{131} \mathrm{I}$ therapy can be considered. While low ${ }^{131} \mathrm{I}$ activities $(1.1 \mathrm{GBq} ; 30 \mathrm{mCi})$ successfully ablate 
normal thyroid tissue remnant located in the thyroidectomy bed $[19,20]$, effective treatment for iodine-avid regional and distant metastatic disease has traditionally required higher ${ }^{131}$ I activities, as demonstrated by lesion dosimetry calculation studies [21-24]. It is important to discuss the study of Castagna et al. [25], which assessed the effectiveness of $1.11 \mathrm{GBq}(30 \mathrm{mCi})$ vs. $3.7 \mathrm{GBq}(100 \mathrm{mCi})$ of I- 131 for the treatment of $225 \mathrm{DTC}$ patients classified as intermediate risk for recurrence. The rates of disease remission (76.5 vs. $72.1 \%$ ), persistent (biochemical or metastatic) disease (18.8 vs. $23.6 \%)$, recurrent disease (2.4 vs. $2.1 \%)$, and of death (2.4 vs. $2.1 \%)$ were not statistically different in patients treated with $1.11 \mathrm{GBq}(30 \mathrm{mCi})$ versus 3.7 $\mathrm{GBq}(100 \mathrm{mCi})$, the authors concluding that "high 131-I activities have no major advantage over low activities." Subsequently, other authors have referenced this article in support of promulgating that "less I-131 is more" for adjuvant treatment [26]. Although not statistically different in the two groups, the rates of persistent and recurrent disease and even death are concerning. This paper has then been used to advance the idea that $30 \mathrm{mCi}$ is as equally effective as $100 \mathrm{mCi}$; however, it could also be argued that it shows that $100 \mathrm{mCi}$ is equally ineffective as $30 \mathrm{mCi}$. The potential bias for prescribing higher RAI activity to patients with more aggressive histopathological features cannot be entirely excluded in this retrospective study. Moreover, the iodine-aviditiy of the metastatic disease was not assessed prior to RAI administration. Therefore, we consider that risk stratification systems need to include imaging information that will clarify the status of regional and/or distant metastatic disease (as present/or absent) and determine the functional nature of metastases (as iodine-avid or noniodine avid).

\section{Does surgical pathology provide reliable information for management decisions?}

Post-operative management, including RAI administration or lack thereof, is predicated on a risk stratification system based on surgical pathology information [4]. However, the surgical pathology information is inherently limited by the extent of surgical dissection, and in many patients information on nodal status is either unavailable since no lymph nodes were excised $(\mathrm{Nx})$, or very limited since the number of lymph nodes submitted for pathologic examination is too low to rule out the presence of occult residual disease after surgery. For example, the probability of falsely identifying a patient as node negative was estimated to be $53 \%$ for patients with a single lymph node (LN) examined; however, this probability decreased to less than $10 \%$ when more than six LNs were pathologically examined. To rule out occult nodal metastatic disease with $90 \%$ confidence, six LNs would need to be submitted for pathologic examination for patients with $\mathrm{T} 1 \mathrm{~b}$ tumors, nine LNs for patients with T2 tumors, and $18 \mathrm{LNs}$ for patients with T3 disease [27]. Most concerning is that lymphadenectomy is often limited or not performed in $50-70 \%$ cases, especially when the patients are a priori considered to belong to the lowor intermediate-risk groups [25].

The central compartment is considered the first tumor drainage territory in most DTC. The role of prophylactic central compartment lymph node dissection has been debated in the literature over the past few decades. Although its routine use increases the accuracy in tumor staging, there is no clear evidence that this procedure leads to a reduction in recurrence or mortality [28]. In order to limit surgical morbidity, many surgeons perform unilateral paratracheal prophylactic lymphadenectomy (including the cervical and upper mediastinal lymph nodes). This approach is acceptable but several points need to be addressed: 1 - There is preferential drainage of cancers located at the upper poles of the thyroid into the ipsilateral lateral compartment (level III) or at the level of the superior thyroid pedicle; 2 - There is preferential drainage of cancers located in the inferior-internal part of the thyroid or in the isthmus into the pretracheal compartment and the delphian lymph node. 3 - Anatomic considerations influence the extent of nodal dissection: the left inferior laryngeal nerve travels along the tracheoesophageal groove and is at low risk of injury during left paratracheal LN dissection; however, the right recurrent laryngeal nerve crosses the inferior thyroid artery and is at a much higher risk of injury during right $\mathrm{LN}$ dissection. Therefore, right paratracheal $\mathrm{LN}$ dissection may be associated with higher rates of nerve injury, or in less experienced hands may lead to incomplete dissection in order to avoid complications. For all these reasons, the information provided by the "prophylactic central lymphadenectomy" might be suboptimal and information regarding the extent of the dissection might also be missing from the operative report. The information can be further limited by the pathological analysis of extirpated nodes, which can vary in completeness and quality between centers.

Therefore, management decisions dictated on risk stratification predicated solely on surgical pathology are inherently limited, leading to incomplete staging and potentially excluding RAI administration for patients that may have benefitted from it had the extend of disease been accurately determined.

\section{Are postsurgical low thyroglobulin levels a reliable marker to rule out RAI administration?}

Post-operative Tg levels cannot be used as a cornerstone criterion to recommend for or against RAI administration. New recommendations include using postsurgical $\mathrm{Tg}$ measurement (either TSH stimulated or nonstimulated, obtained a few weeks after total thyroidectomy and before RAI remnant ablation) as a tool to aid in initial risk stratification and adjuvant RAI therapy decision-making [4]. However, a post-treatment 
RAI whole body scan (post-Rx WBS) demonstrating residual thyroid tissue with a corresponding undetectable serum Tg was reported in up to $20 \%$ of DTC patients [29]. Moreover, up to $6 \%$ of such patients had confirmed loco-regional or distant metastases in addition to thyroid tissue remnants [30]. Most importantly, RAI administration likely contributed to good patient outcome observed in previous studies on the role of preablative $\mathrm{Tg}$, while similar results in non-ablated patients remain unproved.

Different technical problems still affect Tg measurement and need to be carefully considered when using $\mathrm{Tg}$ as a marker for the presence of thyroid tissue. First, interference from $\mathrm{TgAb}$ compromises the use of serum $\mathrm{Tg}$ as a tumor marker in up to $25 \%$ of DTC patients by reducing Tg measurement results in immunometric methods [31]. Second, interferences by heterophilic antibodies, as well as the so-called "high-dose hook effect," or decreased immunological reactivity or changes of the structural conformation of the $\mathrm{Tg}$ molecule may in rare instances lead to report inappropriately low serum $\mathrm{Tg}$ values in sera with high $\mathrm{Tg}$ concentrations [32]. The importance of these interferences has been documented by analyzing sera from 47 DTC patients presenting undetectable serum Tg, but residual ${ }^{131}$ I uptake on a PT-WBS [33]. This study also pointed to the variability between different Tg immunoassays [33].

As explicitly admitted in ATA 2015 guidelines, no precise $\mathrm{Tg}$ value cutoffs are available to define what is an excellent response after surgery justifying RAI omission. Indeed, postoperative serum $\mathrm{Tg}$ value is strongly dependent on the type of determination (stimulated or nonstimulated), type of stimulation (rh-TSH vs. endogenous hypothyroid stimulation), the actual level of TSH stimulation and the volume of remnant thyroid tissue. Therefore, the level of postoperative Tg cannot alone guide the decision for or against RAI administration [29, $30,34]$. It appears that the postoperative serum $\mathrm{Tg}$ value will be more helpful in identifying patients that will benefit from RAI ablation rather than in identifying patients that do not require ablation [4].

All in all, a low or undetectable Tg cannot be considered a completely reliable marker to safely exclude DTC patients from RAI ablation. Therefore, a benchmark between ${ }^{131} \mathrm{I}$ imaging and $\mathrm{Tg}$ measurement remains of pivotal value by authenticating the use of serum Tg during further follow-up of DTC patients [35].

\section{Does postoperative RAI administration improve risk-stratified care management?}

The use of RAI following total thyroidectomy has several distinct advantages: 1 - the ${ }^{131}$ I post-therapy scan (post-Rx WBS) completes post-operative staging, and the information obtained may modify the treatment plan and long-term surveillance, 2 - it provides early reassurance of disease-free status, 3- it facilitates long-term follow-up by reducing concentrations of sensitive Tg below the threshold of detectability, 4- it avoids useless and repetitive costly medical procedures (i.e., rhTSH stimulation, neck US, and FNA biopsies for equivocal US findings).

Imaging-based staging at ablation reveals the true extent of residual disease. Notably, in about $5 \%$ of patients, the ${ }^{131} \mathrm{I}$ whole-body scan has uncovered occult distant spread, mainly pulmonary or skeletal metastases [18, 36-39]. This small percentage of cases has its importance as distant metastases are the main purveyors of death from DTC and early detection on the ${ }^{131} \mathrm{I}$ scan is a favorable situation that offers chances for cure [18, 36-39]. Moreover, the ${ }^{131}$ I scan has substantially improved in recent years; ${ }^{131}$ I SPECT-CT acquisition, which is performed after whole-body scan on the neck area or on suspicious body foci, allows to substantially improve specificity $[40,41] .{ }^{131}$ I SPECT-CT has an excellent reproducibility and high detection sensitivity in neck compartments II, low VI and V. Thus, DTC management may be modified by post-Rx WBS ${ }^{131}$ I imaging in roughly $15 \%$ to $25 \%$ of the patients [42]. Without ablation, a substantial percentage (5-40\%) of these metastatic nodes will persist. [43] Even small or occult malignant lymph nodes are likely to grow slowly with time, causing secondary costs, anxiety for the patients and sometimes worsening of the overall prognosis. Indeed, recent data indicate that microscopic lymph node involvement represents a distinct prognostic entity which is associated with an intermediate outcome between the N0/Nx and the N1 macroscopic patients [44]. Post-operative DxWBS and/or post-Rx WBS evaluation permits early detection and treatment of iodine-avid regional and distant metastases completing staging and risk stratification, provide a baseline for assessing therapeutic response and contribute to long-term follow-up decisions (such as the intensity and duration of L-T4 suppression, frequency of Tg determinations and neck US surveillance).

\section{Does postoperative RAI administration facilitate long-term follow-up and improve dynamic risk stratification systems?}

Information obtained with postoperative RAI administration provides the baseline for assessing the response to initial treatment enabling dynamic risk stratification (DRS), which determines the long-term follow-up strategy. Very early, Mellière et al., has advanced the concept that final staging of patients who received total thyroidectomy cannot be obtained until results from the ${ }^{131}$ I ablation scan is obtained and results from Tg measurement at 6 months post-ablation are available [11]. They called this dynamic stratification "ExtensionThyroglobulin". They identified as the "high-risk group" patients who either have widely invasive tumors (e.g. tumors that invade the larynx, trachea, esophagus, recurrent laryngeal 
nerve or vessels) or distant metastases (discovered initially or on the post-Rx WBS after initial ${ }^{131}$ I ablation) or who have a stimulated Tg level at 6 months of more than $10 \mu \mathrm{g} / \mathrm{L}$ (after T4 withdrawal; $\mathrm{Tg}$ testing in association with a diagnostic ${ }^{131} \mathrm{I}$ whole-body scan and neck ultrasound examination) [11]. The 25-year actuarial rate of cancer-related death for this high risk group was $46.9 \%$. Comparatively, the 25 -year rate of cancer-related death and cancer recurrence with this approach in patients with satisfactory testing were $1.4 \%$ and $3.8 \%$, respectively, in patients with intrathyroidal tumor $(>1 \mathrm{~cm})$ without nodal metastases, and $0 \%$ and $5.3 \%$ in patients with lymph node metastases [11]. Because of its clinical relevance, the concept of dynamic risk stratification achieved impetus and was first formalized as such by Tuttle et al. in 2010 [45]. DRS after total thyroidectomy and ${ }^{131}$ I ablation includes Tg measurement (after stimulation or under levothyroxine, L-T4, therapy, especially with the new sensitive assays) and imaging studies including neck US, and when indicated ${ }^{131}$ I scanning, ${ }^{18}$ FDG PET/CT, or other imaging studies. DRS classifies response to therapy into three categories: excellent, acceptable, or incomplete response [45]. These categories are strongly predictive of the course of further follow-up, especially in the subgroup of "excellent responders" who have a very high rate of permanent remission (i.e. absence of disease) during follow-up. The DRS better identifies the risk of PRD (persistent or relapsed disease) as compared to the ATA/ETA staging systems [46] and appears to be a powerful classifier which can be used to promptly reassure a majority of patients who will not require further specialized tests. However, we must keep in mind that a follow-up of at least 10 years is needed to assess the true rate of persistent or relapsed disease (PRD) occurrence. This holds especially true for low risk patients which have been ablated using the comparatively novel strategy of administering low ${ }^{131}$ I activities. However, this long followup period is far from being met in several high ranking, much touted recent papers [47].

The accuracy of the DRS staging now relies strongly on new $\mathrm{Tg}$ assays with routine sensitivity $<0.1 \mathrm{ngLml}$. After remnant ${ }^{131} \mathrm{I}$ ablation, several publications report basal $\mathrm{Tg}$ concentration values of over $0.3 \mathrm{ng} / \mathrm{mL}$ indicate a significant risk of relapse [48]. Unfortunately, there are no convincing data showing any Tg threshold suggesting PRD in case ${ }^{131} \mathrm{I}$ ablation is not performed. It is likely that none may be proven with sufficient sensitivity and specificity, especially considering that the ability to produce $\mathrm{Tg}$ varies widely between tumors and remnants. In addition, sensitive Tg is often detectable after surgery and may be influenced by variations in TSH levels. Even then, only time will prove whether a patient is really disease-free. In contrast, 6 to 9 months after ${ }^{131}$ I ablation, more than $90 \%$ of the patients have undetectable sensitive Tg levels, making the subsequent follow-up extremely simple and reliable. Therefore, we conclude that patientindividualized post-operative RAI administration based on integration of histopathology, postoperative Tg level and functional imaging information is a reasonable option that simplifies clinical management and long-term follow-up surveillance strategy.

\section{Does adjuvant or therapeutic RAI administration improve clinical outcomes?}

RAI administration is an effective treatment for iodine-avid metastatic disease. The adjuvant therapeutic effect of RAI is highly interesting, especially for destroying small, at most a few millimeters in diameter sized tumor foci, in thyroid remnants or lymph nodes, or eradicating occult distant micrometastases. Many publications report a high frequency of uncovered persistent disease in the neck, mediastinal nodes, or distant spread at ablation. This is rather expected in intermediate and high risk patients, but may occur even in the so called "low-risk" group [13]. In the literature, $5 \%$ to $25 \%$ of patients show unsuspected foci of pathologic uptake using ${ }^{131} \mathrm{I}$ scanning and changes in therapy planning are reported in a significant percentage of cases.

Most published evidence demonstrates a reduced recurrence rate after RAI $[2,8,9]$, despite the presence of some conflicting data (mainly from observational studies). Furthermore, the tumoricidal effect of ${ }^{131} \mathrm{I}$ has been reported to be effective (94\% eliminated lymph node metastases) especially on lymph nodes smaller than $0.9 \mathrm{~mL}$ identified on ${ }^{131} \mathrm{I}$ SPECT-CT [17]. Such interesting studies are less frequently reported possibly because they rely upon the newer ${ }^{131} \mathrm{I}$ SPECT-CT technology and routine pathology confirmation would be difficult to obtain. Nonetheless, the adjuvant effect of ${ }^{131} \mathrm{I}$ is highly probable, resulting in elimination of small residual nodal metastases or in identification of larger structural disease that could potentially justify re-operation. A big concern arises here for many practitioners when looking at RAI decision-making tables of the 2015 ATA Guidelines [4], since recommendations mainly recommend against RAI administration, for instance in $\mathrm{pT} 1 \mathrm{~b}, \mathrm{pT} 2$, and even some $\mathrm{pT} 3$ N0/Nx ATA risk groups. Even in higher risk patients often no explicit recommendation for ${ }^{131} \mathrm{I}$ therapy is given (but simply the term "consider" ${ }^{131}$ I therapy). These recommendations are made in spite of the presence of conflicting observational data. In absence of any ${ }^{131} \mathrm{I}$ administration or only using low activities, the adjuvant and a fortiori therapeutic goals of RAI will be lost in patients with unsuspected persistent metastatic disease foci after surgery $[2,8,9,11]$. Since the literature cannot advise for or against these statements, further long-term follow-up studies are needed before recommendations to change effective clinical practice are accepted as standard of care. As long as the results of such studies are still pending, it would be prudent to refrain from strong positions against ${ }^{131} \mathrm{I}$ where 
thus far the course of disease with ${ }^{131}$ I therapy has been so good [1-3].

As for the prescribed RAI activity, this should be carefully selected. To simply raise as principle "the lower the better" [26], makes little sense. In radiation oncology the proposition that a lesser radiation therapy dose is better would be unacceptable, and the same applies for chemotherapy treatment.

If the goal is only to perform thyroid remnant ablation (i.e. elimination of normal thyroid tissue remnant) in order to facilitate initial post-therapy scanning and dynamic restaging, especially where the assessment of $\mathrm{Tg}$ levels are concerned, then low ${ }^{131}$ I activities provide a practical advantage (limited or no hospital stay with cost reduction) and minimize the risk of side effects, while still achieving high ablation rates. At least 12 prospective studies have shown that an activity of $1110 \mathrm{MBq} / 30 \mathrm{mCi}$ met certain arbitrary endpoints roughly as effectively as the historically often-used $3700 \mathrm{MBq} /$ $100 \mathrm{mCi}$, provided the size of the post-surgically remaining tissue "remnant" is not too large [49]. A few papers have even reported similar ablation rates using $\sim 20 \mathrm{mCi}[34,50]$, while in contrast, some report a significantly lower ablation rate at $30 \mathrm{mCi}$ than when using $100 \mathrm{mCi}$. Most importantly, for patients $\geq 45$ years, the rate of disease recurrence and DTCspecific mortality was found in some studies to be significantly higher in patients treated with lower RAI activity $(\leq 2000 \mathrm{MBq}$ or $54 \mathrm{mCi}$ ), as compared to higher ablative RAI activities [5].

Considering the endpoints of importance, such as rates of persistent and/or recurrent disease, disease-specific death, simplified surveillance and lower costs, the post-operative RAI administration remains a reasonable option. As we fully discussed earlier, the reasons why ${ }^{131} \mathrm{I}$ has been administered for decades goes far beyond the simple aim of "ablation" and, therefore, the activity to be administered should be carefully selected on the basis of the particular clinical situation.

\section{Conclusion}

The temptation to control every situation is strong both in medicine and in society. Guidelines should integrate these normal variations and remain rather open. Apart from that, many endpoints of ${ }^{131}$ I still remain controversial or unknown. Therefore, recommendations for RAI should integrate not only hard oncological criteria, but also tailored therapy management and surveillance, patient's wellbeing, and quality of life, at a lower cost for society. Finally, we strongly believe that for the reasons explained above, RAI remains a valuable adjuvant post-operative treatment for most DTC patients. Although it is always good to question and reevaluate current practice, care should be taken not to discard previous management guidelines on postoperative ${ }^{131} \mathrm{I}$ therapy before proper evidence is produced that management without RAI is as safe and as effective in the selected patient categories as administration of this proven effective therapeutic modality.

The limited space of an editorial allows us to discuss several salient points that only scratch the surface of a flawed approach in the recent ATA Guidelines regarding the contribution of radioiodine imaging and treatment to thyroid cancer management. Taking into consideration the slow progression of thyroid cancer, the adverse clinical consequences of these recommendations may not become apparent until years have passed and we find patients with recurrences that could prove difficult to treat, and could have been avoided.

\section{Compliance with ethical standards}

Funding The review did not receive any specific grant from any funding agency in the public, commercial or not-for-profit sector.

Disclosure of potential conflicts of interest FAV is a consultant to Bayer Healthcare and Sanofi-Genzyme and has accepted Speaker Honoraria from Sanofi-Genzyme and Diasorin. LG has accepted Speaker Honoraria from Sanofi-Genzyme, BRAHMS GmbH and ROCHE Diagnostics.

Ethical approval This article does not contain any studies with human participants or animals performed by any of the authors.

Informed consent This article does not contain any studies with human participants or animals performed by any of the authors.

\section{References}

1. Luster M, Clarke SE, Dietlein M, et al. Guidelines for radioiodine therapy of differentiated thyroid cancer. Eur J Nucl Med Mol Imaging. 2008;35(10):1941-59.

2. Mazzaferri EL, Jhiang SM. Long-term impact of initial surgical and medical therapy on papillary and follicular thyroid cancer. Am J Med. 1994;97(5):418-28.

3. Van Nostrand D. The benefits and risks of I-131 therapy in patients with well-differentiated thyroid cancer. Thyroid. 2009;19(12): 1381-91.

4. Haugen BR, Alexander EK, Bible KC, et al. 2015 American thyroid association management guidelines for adult patients with thyroid nodules and differentiated thyroid cancer: the american thyroid association guidelines task force on thyroid nodules and differentiated thyroid cancer. Thyroid. 2016;26(1):1-133.

5. Verburg FA, Mader U, Reiners C, Hanscheid H. Long-term survival in differentiated thyroid cancer is worse after low-activity initial post-surgical 131I therapy in both high- and low-risk patients. J Clin Endocrinol Metab. 2014;99(12):4487-96.

6. Cooper DS, Doherty GM, Haugen BR, et al. Revised American thyroid association management guidelines for patients with thyroid nodules and differentiated thyroid cancer. Thyroid. 2009;19(11):1167-214.

7. Vaisman F, Shaha A, Fish S, Michael TR. Initial therapy with either thyroid lobectomy or total thyroidectomy without radioactive iodine remnant ablation is associated with very low rates of structural disease recurrence in properly selected patients with differentiated thyroid cancer. Clin Endocrinol (Oxf). 2011;75(1):112-9. 
8. Jonklaas J, Sarlis NJ, Litofsky D, et al. Outcomes of patients with differentiated thyroid carcinoma following initial therapy. Thyroid. 2006;16(12):1229-42.

9. Ruel E, Thomas S, Dinan M, Perkins JM, Roman SA, Sosa JA. Adjuvant radioactive iodine therapy is associated with improved survival for patients with intermediate-risk papillary thyroid cancer. J Clin Endocrinol Metab. 2015;100(4):1529-36.

10. Higashi T, Nishii R, Yamada S, et al. Delayed initial radioactive iodine therapy resulted in poor survival in patients with metastatic differentiated thyroid carcinoma: a retrospective statistical analysis of 198 cases. J Nucl Med. 2011;52(5):683-9.

11. Melliere D, Hindie E, Becquemin JP, Desgranges P, Allaire E, Geachan E. Differentiated thyroid carcinoma-how to improve the long-term results? Twenty-five-year outcomes of 850 patients. Bull Acad Natl Med. 2006;190(1):89-106. discussion -9.

12. Avram AM, Esfandiari NH, Wong KK. Preablation 131-I scans with SPECT/CT contribute to thyroid cancer risk stratification and 131-I therapy planning. J Clin Endocrinol Metab. 2015;100(5): 1895-902.

13. Avram AM, Fig LM, Frey KA, Gross MD, Wong KK. Preablation 131-I scans with SPECT/CT in postoperative thyroid cancer patients: what is the impact on staging? J Clin Endocrinol Metab. 2013;98(3):1163-71.

14. Chen MK, Yasrebi M, Samii J, Staib LH, Doddamane I, Cheng DW. The utility of I-123 pretherapy scan in I-131 radioiodine therapy for thyroid cancer. Thyroid. 2012;22(3):304-9.

15. Silberstein EB. Comparison of outcomes after (123)I versus (131)I pre-ablation imaging before radioiodine ablation in differentiated thyroid carcinoma. J Nucl Med. 2007;48(7):1043-6.

16. Wong KK, Sisson JC, Koral KF, Frey KA, Avram AM. Staging of differentiated thyroid carcinoma using diagnostic 131I SPECT/CT. AJR Am J Roentgenol. 2010;195(3):730-6.

17. Schmidt D, Linke R, Uder M, Kuwert T. Five months' follow-up of patients with and without iodine-positive lymph node metastases of thyroid carcinoma as disclosed by (131)I-SPECT/CT at the first radioablation. Eur J Nucl Med Mol Imaging. 2010;37(4):699-705.

18. Durante C, Haddy N, Baudin E, et al. Long-term outcome of 444 patients with distant metastases from papillary and follicular thyroid carcinoma: benefits and limits of radioiodine therapy. J Clin Endocrinol Metab. 2006;91(8):2892-9.

19. Schlumberger M, Catargi B, Borget I, et al. Strategies of radioiodine ablation in patients with low-risk thyroid cancer. N Engl J Med. 2012;366(18):1663-73.

20. Mallick U, Harmer C, Yap B, et al. Ablation with low-dose radioiodine and thyrotropin alfa in thyroid cancer. N Engl J Med. 2012;366(18):1674-85.

21. Maxon 3rd HR, Englaro EE, Thomas SR, et al. Radioiodine-131 therapy for well-differentiated thyroid cancer - a quantitative radiation dosimetric approach: outcome and validation in 85 patients. $\mathrm{J}$ Nucl Med. 1992;33(6):1132-6.

22. Maxon HR, Thomas SR, Samaratunga RC. Dosimetric considerations in the radioiodine treatment of macrometastases and micrometastases from differentiated thyroid cancer. Thyroid. 1997;7(2):183-7.

23. Freudenberg LS, Jentzen W, Petrich T, et al. Lesion dose in differentiated thyroid carcinoma metastases after rhTSH or thyroid hormone withdrawal: 124I PET/CT dosimetric comparisons. Eur J Nucl Med Mol Imaging. 2010;37(12):2267-76.

24. Sgouros G, Hobbs RF, Atkins FB, Van Nostrand D, Ladenson PW, Wahl RL. Three-dimensional radiobiological dosimetry (3D-RD) with 124I PET for 131I therapy of thyroid cancer. Eur J Nucl Med Mol Imaging. 2011;38 Suppl 1:S41-7.

25. Castagna MG, Cevenini G, Theodoropoulou A, et al. Post-surgical thyroid ablation with low or high radioiodine activities results in similar outcomes in intermediate risk differentiated thyroid cancer patients. Eur J Endocrinol. 2013;169(1):23-9.
26. Pacini F. Radioactive iodine activities for postsurgical thyroid ablation: the lower the better. Eur Thyroid J. 2013;1(4):213-5.

27. Robinson TJ, Thomas S, Dinan MA, Roman S, Sosa JA, Hyslop T. How many lymph nodes are enough? assessing the adequacy of lymph node yield for papillary thyroid cancer. J Clin Oncol. 2016;34(28):3434-9.

28. Lang BH, Ng SH, Lau LL, Cowling BJ, Wong KP, Wan KY. A systematic review and meta-analysis of prophylactic central neck dissection on short-term locoregional recurrence in papillary thyroid carcinoma after total thyroidectomy. Thyroid. 2013;23(9): 1087-98.

29. Cherk MH, Francis P, Topliss DJ, Bailey M, Kalff V. Incidence and implications of negative serum thyroglobulin but positive I-131 whole-body scans in patients with well-differentiated thyroid cancer prepared with rhTSH or thyroid hormone withdrawal. Clin Endocrinol. 2012;76(5):734-40.

30. Park EK, Chung JK, Lim IH, et al. Recurrent/metastatic thyroid carcinomas false negative for serum thyroglobulin but positive by posttherapy I-131 whole body scans. Eur J Nucl Med Mol Imaging. 2009;36(2):172-9.

31. Spencer C, Fatemi S. Thyroglobulin antibody (TgAb) methods strengths, pitfalls and clinical utility for monitoring TgAb-positive patients with differentiated thyroid cancer. Best Pract Res Clin Endocrinol Metab. 2013;27(5):701-12.

32. Giovanella L, Clark PM, Chiovato L, et al. Thyroglobulin measurement using highly sensitive assays in patients with differentiated thyroid cancer: a clinical position paper. Eur J Endocrinol. 2014;171(2):R33-46.

33. Giovanella L, Suriano S, Ceriani L, Verburg FA. Undetectable thyroglobulin in patients with differentiated thyroid carcinoma and residual radioiodine uptake on a postablation whole-body scan. Clin Nucl Med. 2011;36(2):109-12.

34. Clerc J, Bienvenu-Perrard M, de Malleray CP, et al. Outpatient thyroid remnant ablation using repeated low 131-iodine activities $(740 \mathrm{MBq} / 20 \mathrm{mCix} 2)$ in patients with low-risk differentiated thyroid cancer. J Clin Endocrinol Metab. 2012;97(3):871-80.

35. Giovanella L, Verburg FA. Ruling out 131I ablation in low-risk differentiated thyroid carcinoma basing on thyroglobulin measurement. Thyroid. 2011;21(7):809-10. author reply 11-2.

36. Hindie E, Melliere D, Lange F, et al. Functioning pulmonary metastases of thyroid cancer: does radioiodine influence the prognosis? Eur J Nucl Med Mol Imaging. 2003;30(7):974-81.

37. Hindie E, Zanotti-Fregonara P, Keller I, et al. Bone metastases of differentiated thyroid cancer: impact of early 131I-based detection on outcome. Endocr Relat Cancer. 2007;14(3):799-807.

38. Casara D, Rubello D, Saladini G, et al. Different features of pulmonary metastases in differentiated thyroid cancer: natural history and multivariate statistical analysis of prognostic variables. J Nucl Med. 1993;34(10):1626-31.

39. Orita Y, Sugitani I, Matsuura M, et al. Prognostic factors and the therapeutic strategy for patients with bone metastasis from differentiated thyroid carcinoma. Surgery. 2010;147(3):424-31.

40. Glazer DI, Brown RK, Wong KK, Savas H, Gross MD, Avram AM. SPECT/CT evaluation of unusual physiologic radioiodine biodistributions: pearls and pitfalls in image interpretation. Radiographics :Rev Publ Radiol Soc N Am Inc. 2013;33(2):397418.

41. Xue YL, Qiu ZL, Song HJ, Luo QY. Value of (1)(3)(1)I SPECT/CT for the evaluation of differentiated thyroid cancer: a systematic review of the literature. Eur J Nucl Med Mol Imaging. 2013;40(5): 768-78.

42. Schmidt D, Szikszai A, Linke R, Bautz W, Kuwert T. Impact of 131I SPECT/spiral CT on nodal staging of differentiated thyroid carcinoma at the first radioablation. J Nucl Med. 2009;50(1):18-23.

43. Patron V, Hitier M, Bedfert C, Le Clech G, Jegoux F. Occult lymph node metastases increase locoregional recurrence in differentiated 
thyroid carcinoma. Ann Otol Rhinol Laryngol. 2012;121(5):28390.

44. Bardet S, Ciappuccini R, Quak E, et al. Prognostic value of microscopic lymph node involvement in patients with papillary thyroid cancer. J Clin Endocrinol Metab. 2015;100(1):132-40.

45. Tuttle RM, Tala H, Shah J, et al. Estimating risk of recurrence in differentiated thyroid cancer after total thyroidectomy and radioactive iodine remnant ablation: using response to therapy variables to modify the initial risk estimates predicted by the new American thyroid association staging system. Thyroid. 2010;20(12):1341-9.

46. Cano-Palomares A, Castells I, Capel I, et al. Response to initial therapy of differentiated thyroid cancer predicts the long-term outcome better than classical risk stratification systems. Int $\mathrm{J}$ Endocrinol. 2014;2014:591285.
47. Blumhardt R, Wolin EA, Phillips WT, et al. Current controversies in the initial post-surgical radioactive iodine therapy for thyroid cancer: a narrative review. Endocr Relat Cancer. 2014;21(6):R473-84.

48. Brassard M, Borget I, Edet-Sanson A, et al. Long-term follow-up of patients with papillary and follicular thyroid cancer: a prospective study on 715 patients. J Clin Endocrinol Metab. 2011;96(5):1352-9.

49. Cheng W, Ma C, Fu H, et al. Low- or high-dose radioiodine remnant ablation for differentiated thyroid carcinoma: a meta-analysis. J Clin Endocrinol Metab. 2013;98(4):1353-60.

50. Caglar M, Bozkurt FM, Akca CK, et al. Comparison of 800 and $3700 \mathrm{MBq}$ iodine-131 for the postoperative ablation of thyroid remnant in patients with low-risk differentiated thyroid cancer. Nucl Med Commun. 2012;33(3):268-74. 